\title{
The influence of antenatal exposure to phthalates on subsequent female reproductive development in adolescence: a pilot study
}

\author{
Roger Hart ${ }^{1}$, Dorota A Doherty ${ }^{1,2}$, Hanne Frederiksen ${ }^{3}$, Jeffrey A Keelan ${ }^{1,2}$, Martha Hickey ${ }^{4}$, \\ Deborah Sloboda $^{5}$, Craig E Pennell ${ }^{1,2}$, John P Newnham ${ }^{1,2}$, Niels E Skakkebaek ${ }^{3}$ and \\ Katharina M Main ${ }^{3}$ \\ ${ }^{1}$ School of Women's and Infants' Health, University of Western Australia, King Edward Memorial Hospital, 374 Bagot \\ Road, Subiaco, Perth, Western Australia 6008, Australia, ${ }^{2}$ Women and Infants Research Foundation, King Edward \\ Memorial Hospital, Perth, Western Australia, Australia, ${ }^{3}$ University Department of Growth and Reproduction, \\ Rigshospitalet, Copenhagen, Denmark, ${ }^{4}$ Department of Obstetrics and Gynaecology, University of Melbourne, \\ Melbourne, Victoria, Australia and ${ }^{5}$ Department of Biochemistry and Biomedical Sciences, McMaster University, \\ Hamilton, Ontario, Canada L8S 4K1
}

Correspondence should be addressed to R Hart; Email: roger.hart@uwa.edu.au

\begin{abstract}
We hypothesised that antenatal exposure to ubiquitous phthalates may lead to an earlier menarche and a lower prevalence of polycystic ovarian syndrome (PCOS) and polycystic ovarian morphology (PCO) in adolescence. The Western Australian Pregnancy Cohort (Raine) Study recruited 3000 women at 18 weeks of gestation in 1989-1991, 1377 had antenatal serum stored without thawing at $-80{ }^{\circ} \mathrm{C}$. An unselected subset was evaluated in the early follicular phase for PCO and PCOS by ultrasound and serum evaluation in adolescence. Serum was analysed for anti-Müllerian hormone (AMH), inhibin B, sex hormone binding globulin (SHBG), testosterone, androstenedione and DHEAS. Four hundred microlitres of the frozen maternal serum underwent isotope-diluted liquid chromatography-tandem mass spectrometry, with preceding enzymatic deconjugation followed by solid-phase extraction to determine phthalate exposure.

Two hundred and forty four girls attended assessment and most common phthalate metabolites were detectable in the majority of the 123 samples available. Several phthalates were negatively associated with maternal SHBG, and associations with maternal androgens were less consistent. The sum of the metabolites of di-(2-ethylhexyl) phthalate was associated with a non-significant tendency towards an earlier age at menarche $(P=0.069)$. Uterine volume was positively associated with mono-(carboxy-iso-octyl) phthalate $(P=0.018)$. Exposure to monoethyl phthalate (MEP) and the sum of all phthalate metabolites ( (all phth.m) were protective against PCOS in adolescence $(P=0.001$ and $P=0.005$ respectively). There were negative associations of MEP with $P C O(P=0.022)$ and of $M E P$ with serum AMH $(P=0.031)$. Consequently, our data suggest that antenatal exposure to environmental phthalates may be associated with oestrogenic and/or anti-androgenic reproductive effects in adolescent girls.

Reproduction (2013) 147 379-390
\end{abstract}

\footnotetext{
This paper forms part of a special issue of Reproduction on Endocrine Disrupters. This article was presented at the 7 th Copenhagen Workshop on Endocrine Disrupters, 28-31 May 2013. The meeting was supported by the Danish Ministry of the Environment - Environmental Protection Agency as an activity under the Danish Centre on Endocrine Disrupters. Publication of this special issue has been supported by the Society for Reproduction and Fertility. The opinions or views expressed in this special issue are those of the authors, and do not necessarily reflect the opinions or recommendations of the Danish Ministry of the Environment - Environmental Protection Agency or the Society for Reproduction and Fertility. The Guest Editors for this special issue were Anna-Maria Andersson, Hanne Frederiksen, Niels Erik Skakkebæk, Rigshospitalet, Denmark, Kenneth M Grigor, Western General Hospital, Edinburgh, UK and Jorma Toppari, University of Turku, Finland.
}

\section{Introduction}

There is an increasing awareness among the scientific community that environmental factors may adversely affect human reproductive health. It has been reported, although also disputed, that sperm counts have decreased and the incidence of undescended testis, hypospadias and testicular cancer has increased in some countries (Handelsman 2001, Skakkebaek et al. 2001, 2007, Andersson et al. 2008, Toppari et al. 2010). Strong evidence exists from animal models that exposure to endocrine disrupters can exert adverse effects on reproductive outcomes in the offspring. Some pesticides 
and phthalates have the potential to cause cryptorchidism and decreased sperm production (Pierik et al. 2004).

In females, antenatal exposure of sheep and monkeys to androgens has been shown to cause a polycystic ovarian syndrome (PCOS)-like phenotype, although this as a potential cause of PCOS and is highly speculative (Abbott et al. 2005). Furthermore, there is evidence for a direct effect of some pesticides on ovarian function. The pesticide metabolite HPTE (2,2-bis-(p-hydroxyphenyl)1,1,1-trichloroethane) accelerates anti-Müllerian hormone $(\mathrm{AMH})$ production and interferes with folliculogenesis (Uzumcu et al. 2006). Thus, exposure of the human developing ovary to endocrine disrupting chemicals at the time of sex differentiation may potentially lead to changes in pubertal timing (Massart et al. 2006) and reproductive disorders later in life (Skakkebaek et al. 2001).

Diesters of phthalic acid (phthalates) are ubiquitous within our environment and are widely used in industrial and personal care products, plastics, paints and some pesticide formulations. Exposure occurs through ingestions, dermal absorption and inhalation (www.atsdr.cdc. gov). Phthalates are recognised to be endocrine disrupting chemicals with anti-androgenic properties (Lyche et al. 2009). They cross the placenta to the developing fetus (Mose et al. 2007, Adibi et al. 2010) and are detectable in amniotic fluid (Huang et al. 2009, Wittassek et al. 2009). Post-natally, they can reach the newborn via breast milk (Main et al. 2006a). Thus, exposure has been shown to be continuous throughout life (Lyche et al. 2009). Absorption of phthalates may be transdermal (Janjua et al. 2008), oral or via inhalation (Hsu et al. 2012); once absorbed, phthalates are rapidly distributed throughout the body and metabolised (Frederiksen et al. 2007).

Phthalates may exert anti-androgenic actions by interfering with steroidogenesis (Auharek et al. 2010, Christiansen et al. 2010, MacLeod et al. 2010, Boberg et al. 2011) and have been associated with a decrease in infant testosterone concentrations and an increase in luteinising hormone and sex hormone binding globulin (SHBG) levels (Main et al. 2006a). Evidence for the reproductive effect of antenatal exposure to phthalates in humans is still sparse (Jurewicz \& Hanke 2011). However, in males, there are reports of a decrease in the anogenital distance (a marker of androgen action) in male infants exposed to higher levels of monoethyl phthalate (MEP), mono- $n$-butyl phthalate (MnBP), monobenzyl phthalate $(\mathrm{MBz} P)$ and mono-iso-butyl phthalate (MiBP) in the antenatal period, as measured from maternal urine during the second trimester of pregnancy (Swan et al. 2005). These data concur with a Japanese study (Suzuki et al. 2012), which reported a reduced anogenital distance in the male offspring of women with higher antenatal urinary levels of mono-(2-ethylhexyl) phthalate (MEHP); however, it was not confirmed in another study (Huang et al. 2009). In spite of this, the effect of some phthalates on steroidogenesis within the fetal human testis has recently been questioned (Mitchell et al. 2012). No changes in testosterone levels were found after di-butyl phthalate (DBP) exposure of fetal testis xenografts. However, in vitro studies using the adult human testis have found associations between phthalate exposure and alterations in steroidogenesis within the testis, which indicates differences in vulnerability between the fetal and adult testis with respect to this endpoint (Desdoits-Lethimonier et al. 2012, Yuan et al. 2012). To date, there are limited data as to the influence of phthalates on female reproductive development other than an association with the delayed development of pubic hair in girls (Frederiksen et al. 2012) and potential associations with precocious puberty and premature thelarche (Jurewicz \& Hanke 2011).

We hypothesised that girls antenatally exposed to antiandrogenic effects by phthalates would have an earlier menarche and a reduction in the prevalence of PCOS. Antenatal androgen exposure is potentially linked to disordered folliculogenesis (Hart et al. 2010a, 2010b) and PCOS in adulthood (Abbott et al. 2005), although this has not been confirmed (Hickey et al. 2009).

To test this hypothesis, we analysed concentrations of phthalate metabolites in maternal blood samples derived from the Western Australian Pregnancy Cohort (Raine) Study to correlate levels with markers of reproductive function in the daughters. After exposure, phthalate diesters are rapidly hydrolysed to monoesters and some of the more non-polar metabolites are further oxidised and/or glucuronidated (Frederiksen et al. 2007). Thus, the phthalate monoesters of nine commonly used phthalate diesters (diethyl phthalate (DEP), the two dibutyl phthalate isomers (DiBP and DnBP), butylbenzyl phthalate (BBzP), di-n-pentyl phthalate (DPP), di-(2-ethylhexyl) phthalate (DEHP), dioctyl phthalate (DOP), di-iso-nonyl phthalate (DiNP) and di-iso-decyl phthalate (DiDP)) together with the carboxylated phthalate metabolites of DEHP and DiNP were analysed in maternal serum samples.

\section{Materials and methods}

The Western Australian Pregnancy Cohort (Raine) Study (www.rainestudy.org.au) was designed to measure the relationships between early life events and subsequent health and behaviour (Macdonald et al. 1996). The study recruited nearly 3000 women at 18 weeks of gestation in Western Australia in 1989-1991 who delivered 2868 live born children (Macdonald et al. 1996). Detailed measurements of fetal growth by ultrasound examination and maternal blood were stored for 1377 randomly selected pregnancies at 18, 24, 28 and 34/36 weeks and again at 38 weeks. A maternal history of cigarette smoking was collected prospectively at 18 and 34/36 weeks. Surveys at age 1, 2, 6, 8, 9, 10, 14 and $16 / 17$ years collected detailed anthropometric, physical fitness, cardiovascular, respiratory, metabolic, endocrine, 
psychological, behavioural, neurocognitive and social measurements. The current cohort includes over 1800 active individuals aged 20-22 years. This is one of the largest and most closely followed prospective cohorts of pregnancy, childhood and adolescence in the world. An unselected subset of 230 girls, the majority of whom were at least 2 years post-menarche, was evaluated as part of an assessment of reproductive function. Age at menarche had previously been prospectively determined by returning a questionnaire at the onset of menarche (Sloboda et al. 2007). The study was approved by the Raine Study Executive Committee and Human Research Ethics Committee of Princess Margaret Hospital. Adolescent subjects and their accompanying parent or guardian provided written consent.

All adolescent girls aged 14-16 years within the cohort were invited to participate in the study. The study visit was scheduled for days 2-5 of their menstrual cycle. This ensured that subjects with both regular and irregular cycles were assessed during the early follicular phase. All visits were timed between 1530 and $1630 \mathrm{~h}$.

\section{Maternal and adolescent blood samples}

Serum was collected from maternal blood that was taken at 18 and 34/36 weeks of gestation and stored in aliquots without thawing at $-80{ }^{\circ} \mathrm{C}$. Samples from a thawed aliquot from both time points from 123 individuals were pooled for analysis of phthalate metabolites to provide an approximation of antenatal phthalate exposure across gestation. As phthalates are non-persistent chemicals, exposure can vary from day to day, but individuals tend to follow their trajectories (Frederiksen et al. 2011). For analysis, the pooled aliquot $(400 \mu \mathrm{l})$ was couriered frozen to Copenhagen, Denmark. Plasma was collected from adolescent daughters on the day of the ultrasound visit and stored at $-80{ }^{\circ} \mathrm{C}$ prior to endocrine analysis.

\section{Pilot study}

Prior to the analysis of all maternal serum samples, two pilot studies were performed to ensure that sampling and storage of maternal serum samples in 1990-1991 did not invalidate the findings. In the first pilot study, the stability of the phthalate metabolites in serum was tested. Serum samples from ten individuals were collected and immediately aliquoted and stored at $-20{ }^{\circ} \mathrm{C}$ with or without addition of phosphoric acid, which is currently considered the best practice in order to inactivate serum enzymes that may hydrolyse diester phthalates into monoesters. Phthalate diesters may contaminate the sample during blood sampling, storage and analysis (Hines et al. 2009). Some aliquots were kept at room temperature for $4 \mathrm{~h}$ before acid addition and storage. Samples were then analysed after 1, 5 and 15 weeks. Samples stored without acid had phosphoric acid added after thawing. Fifteen phthalate metabolites were analysed as described previously (Frederiksen et al. 2010).

In the second pilot study, samples from ten mother-daughter pairs (maternal serum and cord blood) from the Raine Study cohort were analysed for the content of phthalate metabolites to ensure that it was possible to detect these chemical residues in serum after 20 years of storage at $-80^{\circ} \mathrm{C}$.

\section{Phthalate measurements}

Maternal serum samples $(n=123)$ were analysed for total content of 11 phthalate metabolites: MEP, MnBP, MiBP, MBzP, mono-n-pentyl phthalate (MPP), MEHP, mono(2-ethyl-5carboxypentyl) phthalate (MECPP), mono-octyl phthalate (MOP), mono-iso-nonyl phthalate (MiNP), mono-carboxyiso-nonyl phthalate (MCiOP) and mono-iso-decyl phthalate (MiDP) by isotope-diluted liquid chromatography-tandem mass spectrometry (LC-MS/MS), with preceding enzymatic deconjugation followed by solid-phase extraction. The method for preparation of samples, standard solutions and quality controls as well as the instrumental analysis and general method validation was described previously (Frederiksen et al. 2010) and was used with the following modifications: after thawing, $36 \mu \mathrm{l} 1.2 \mathrm{M}$ phosphoric acid was added to $364 \mu \mathrm{l}$ serum and kept at room temperature for $30 \mathrm{~min}$. The final step in the solvent programming for the LC-MS/MS method was changed to $100 \%$ solvent $\mathrm{B}(0.1 \%$ acetic acid in acetonitrile) from 24.1 to $28.0 \mathrm{~min}$, which extended the retention times of the analytes from about 0.1 to $1.0 \mathrm{~min}$.

Samples were analysed in three batches over a period of 1 week. Each batch included standards for calibration curves, 40-45 unknown samples, two blanks, two serum pool controls and two serum pool controls spiked with phthalates standards at a low level. The inter/intra-day variation, expressed as the relative S.D., was below 13\% for most of the analytes, except MiBP (27\%), MPP (30\%), MOP $(23 \%)$ and $\mathrm{MiDP}(31 \%)$. The recovery of spiked control samples was above $93 \%$ for all analytes except MOP (82\%). The limit of detection (LOD) was determined as described previously (Frederiksen et al. 2010).

MEHP and MECPP were combined and expressed as the sum of DEHP metabolites ( $\Sigma$ DEHPm) and MiNP and MCiOP were combined and expressed as the sum of DiNP metabolites ( $\mathrm{D}$ iNPm). In order to combine the metabolites, the sum of each metabolite expressed in molar concentration was multiplied with their respective parent molecular weight (MW) as for instance:

$$
\sum \mathrm{DEHPm}=\left(\frac{C_{\mathrm{MEHP}}}{\mathrm{MW}_{\mathrm{MEHP}}}+\frac{C_{\mathrm{MECPP}}}{\mathrm{MW}_{\mathrm{MECPP}}}\right) \times \mathrm{MW}_{\mathrm{DEHP}}
$$

where $C$ is the measured concentration $(\mathrm{ng} / \mathrm{ml})$ and $\mathrm{MW}$ is the molecular weight $(\mathrm{ng} / \mathrm{nmol})$. Furthermore, the molar sum of all metabolites was combined ( $\Sigma$ all phth.m) and expressed as MEHP by multiplying with the MW of MEHP according to a previously described method (Wolff et al. 2010):

$$
\sum \text { all phth.m }=\left(\frac{C_{\mathrm{m} 1}}{\mathrm{MW}_{\mathrm{m} 1}}+\frac{C_{\mathrm{m} 2}}{\mathrm{MW}_{\mathrm{m} 2}}+\cdots\right) \times \mathrm{MW}_{\mathrm{MEHP}}
$$

where $C_{\mathrm{m} 1}, C_{\mathrm{m} 2}$, etc. are the measured concentrations $(\mathrm{ng} / \mathrm{ml})$ of all the metabolites (MEP, MiBP, MnBP, MBzP, MEHP, MECPP, MiNP and MCiOP) and $M_{\mathrm{m} 1}, M_{\mathrm{m} 2}$, etc. are their respective $M W_{s}(\mathrm{ng} / \mathrm{nmol})$. 


\section{Measurement of uterine and ovarian volume in adolescence}

Transabdominal ultrasound to assess uterine and ovarian volumes was performed with a $5.2 \mathrm{MHz}$ transducer (IU22 Philips Medical Systems, Bothell, WA, USA) or $4 \mathrm{MHz}$ transducer (Voluson 730 Expert, General Electric, Milwaukee, WI, USA), with a standardised measurement protocol as described previously (Hart et al. 2009). The number and diameter of ovarian follicles were recorded. Antral follicles were defined as follicles $<10 \mathrm{~mm}$ in diameter, including all follicles between 2 and $9 \mathrm{~mm}$. The uterine and ovarian volumes were estimated using the formula $0.523 \times$ length $\times$ width $\times$ height of the organ (Adams et al. 1985, Orsini et al. 1985). Polycystic ovarian appearance of the ovaries (polycystic ovarian morphology (PCO)) was defined according to standard international criteria as $\geq 1$ ovary more than $10 \mathrm{~cm}^{3}$ or $\geq 12$ follicles between 2 and $9 \mathrm{~mm}$ in diameter, and if a follicle $\geq 10 \mathrm{~mm}$ was seen, the ultrasound was repeated in the early follicular phase of the next cycle (Balen et al. 2003). The ultrasonographers performing the assessment of ovarian morphology were blind to the results of the phthalate measurements.

\section{Diagnosis of PCOS}

The diagnosis of PCOS was ascertained using the Rotterdam consensus statement (ESHRE/ASRM 2004a, 2004b), as the presence of two of three criteria: PCO morphology, clinical or biochemical hyperandrogenism, or oligo-anovulation. The NIH criteria of PCOS (Zawadzki \& Dunaif 1992) were considered to have been met if menstrual cycles were oligoanovulatory, together with either clinical or biochemical hyperandrogenism (Hickey et al. 2009).

\section{Hormone measurements}

SHBG (68562, Orion Diagnostica, Espoo, Finland), DHEAS (DSL-2700, Beckman, Melbourne, Australia), androstenedione (A4) (DSL-4200, Beckman) and total testosterone (TT; DSL4100, Beckman) were determined in adolescent samples by commercially available immunoassays as described previously (Hickey et al. 2009). Free testosterone concentrations (cFT) were calculated from the measured TT and SHBG as described previously (Hickey et al. 2009). The free androgen index is the TT $(\mathrm{nM}) / \mathrm{SHBG}(\mathrm{nM}) \times 100$.

$\mathrm{AMH}$, follicular stimulating hormone $(\mathrm{FSH})$ and inhibin $\mathrm{B}$ were measured as described previously (Hart et al. 2009). AMH concentrations in adolescent samples were determined by ELISA (A16507; Immunotech - Beckman Coulter). Plasma pools with means of 12 and $77 \mathrm{pmol} / \mathrm{l}$ were used to compare the assay characteristics among the seven assays used for this study. The intra-assay coefficients of variation (CV) were $<5 \%$ and the inter-assay $\mathrm{CV}$ values were $<9 \%$. The assay sensitivity was $1 \mathrm{pmol} / \mathrm{l}$. Inhibin B was determined by ELISA (DSL-10-84100; Beckman) with a sensitivity of $7 \mathrm{pg} / \mathrm{ml}$, intra-assay CV of $9.2 \%$ and inter-assay $\mathrm{CV}$ of $7.0 \%$. FSH concentrations were determined using a Coat-a-Count IRMA from Siemens (Bayswater, Victoria, Australia). FSH sensitivity was $0.75 \mathrm{IU} / \mathrm{I}$ and the intraand inter-assay $\mathrm{CV}$ values were 4.0 and $7.8 \%$ respectively.

\section{Statistical analysis}

Continuous data were summarised using means and S.D. or medians and interquartile ranges (IQRs) according to data normality. Categorical data were summarised using frequency distributions.

For the analysis, all phthalate concentrations $<$ LOD were set to LOD $/ \sqrt{ } 2$. Log transformations of all phthalate measurements and of the reproductive continuous data, when required, were performed to achieve data normality. Phthalate concentrations were also categorised into quartiles. Linear correlation was used to examine the associations between the phthalates and between the phthalates and other continuous reproductive outcomes such as uterine volume and antral follicle count. Correlations between phthalates were estimated using the logtransformed measurements, and correlations between reproductive outcomes and phthalates were obtained using phthalate quartiles. Comparisons of phthalate concentrations between groups of participants such as those with and without the diagnosis of PCOS or presence/absence of PCO morphology were conducted using $t$-tests or Mann-Whitney $U$ tests. When statistically significant associations between phthalates and reproductive outcomes were found, subset analyses on Caucasian participants were performed and these statistically significant associations remained (data not shown).

All hypothesis tests were two-sided and $P$ values $<0.05$ were considered statistically significant. SPSS (version 18.0, SPSS, Inc.) Statistical Software was used for data analysis.

\section{Results}

A total of 244 adolescent girls consented to participate in the study and attend their scheduled assessment on days 2-5 of their menstrual cycle. Twelve girls were using the combined oral contraceptive pill and were excluded from the analysis of ovarian function. The great majority (221, 90.6\%) were Caucasian (including 11 non-Caucasian participants: eight of Asian, two of African and of one South American descent). Of those girls that attended assessment, 123 prenatal maternal serum samples were available, and after exclusions, 121 were included in the analysis. Subject characteristics are shown in Table 1.

\section{Phthalate exposure measurements: pilot studies}

The first pilot study did not show any evidence of instability of serum samples during storage for up to 15 weeks at $-20{ }^{\circ} \mathrm{C}$ or of changes in recovery when processed without acid addition before storing. The second pilot study showed that several of the phthalate metabolites were detectable in the 'old' cohort samples (ten pairs of maternal and cord blood). In both pilot studies, the carboxylated metabolites MECPP and MCiOP were detectable in almost all samples; while the other oxidised metabolites of DEHP and DiNP, the hydroxy- and oxo-form of MEHP and MiNP, only were measurable in very few. We decided to use the 
Table 1 Characteristics of the study population.

\begin{tabular}{lccc}
\hline Characteristic & $\boldsymbol{n}$ total & Median/ & $\begin{array}{c}\text { (Quartiles 1-3, } \\
\text { min-max)/\% }\end{array}$ \\
\hline Age (years) & 121 & 15.1 & $(14.9-15.4 ; 13.3-17.6)$ \\
Age at menarche (years) & 121 & 12.4 & $(11.6-13.2 ; 9.0-16.1)$ \\
Years since menarche & 121 & 2.7 & $(1.9-3.5 ; 0.3-6.0)$ \\
Current BMI & 120 & 22.0 & $(20.0-24.5 ; 16.8-40.8)$ \\
Current BMI ${ }^{\text {group }}$ & 120 & & 72.5 \\
$\quad$ Normal & & 87 & 16.7 \\
Overweight & & 20 & 10.8 \\
Obese & 114 & 13 & 11.4 \\
NIH PCOS & 116 & 32 & 27.6 \\
Rotterdam PCOS & 119 & 41 & 34.5 \\
PCO morphology & 107 & 24 & 22.4 \\
High androgens & 119 & 6.5 & $(4.9-8.5 ; 0.9-26.9)$ \\
Mean ovarian & & & \\
$\quad$ volume $\left(\mathrm{cm}^{3}\right)$ & 120 & 37.8 & $(31.4-50.2 ; 18.3-106.3)$ \\
Uterine volume $(\mathrm{ml})$ & 119 & 11 & $(7-14 ; 0-40)$ \\
Antral follicle count & &
\end{tabular}

aMl; international definition of overweight and obesity according to gender and age by Cole et al. (2000). ' $\mathrm{NIH} /$ Rotterdam PCOS,

polycystic ovary syndrome based on NIH diagnostic criteria/Rotterdam criteria (Zawadzki \& Dunaif 1992, ESHRE/ASRM 2004a, 2004b).

${ }^{\mathrm{c}}$ One or both ovaries $>10 \mathrm{~cm}^{3}$ or $\geq 12$ follicles $2-9 \mathrm{~mm}$.

${ }^{\mathrm{d}} \mathrm{High}$ androgen defined as free testosterone concentration above $\geq 24.45 \mathrm{pmol} / \mathrm{l}$ (Hickey et al. 2011).

carboxylated metabolites as quantitative markers for DEHP and DiNP exposure and thus excluded the other secondary metabolites from the overall analysis, as the other common urinary secondary metabolites of DEHP and DiNP (their hydroxy- and oxo-forms of MEHP and MiNP) were only measurable in very low amounts and in very few of the serum samples.

\section{Phthalate exposure measurements: main study}

MEP, MiBP, MnBP, MBzP, MECPP and MCiOP concentrations were measurable in almost all samples and MEHP was measured in 77 of 123, while MPP, MOP, MiNP and MiDP only were detected in very few samples in concentrations near the detection limits (Table 2). Thus, MPP, MOP and MiDP were not included in the outcome analyses, while MiNP was included in analyses in combination with MCiOP as $\Sigma$ DiNPm.

Most phthalate metabolites, except MEP, were significantly positively correlated with each other (Table 3 ). Despite MEHP and MECPP both being metabolites of DEHP, a non-significant correlation was observed between these metabolites, as 46 of the 123 samples had MEHP concentrations below the LOD (Table 2).

\section{Phthalates and reproductive characteristics in adolescent daughters}

There were no correlations between antenatal serum phthalate concentrations of all metabolites and an earlier age at menarche, except for $\Sigma$ DEHPm concentrations where this inverse relationship approached statistical significance $(r=-0.17, P=0.069)$. The median age of menarche within this group was earlier (mean 12.4 years, IQR 11.6-13.3 years) than the female members of the wider cohort 13.0 years (IQR 12.2-14.2).

$\mathrm{MCiOP}$ was positively associated with uterine volume univariately $(r=0.22, P=0.018)$ and after adjustment for time since menarche $(r=0.22, P=0.024)$. Correlations between uterine volume and MEP, MiBP, MnBP, MEHP, MECPP, $\Sigma \mathrm{MBP}_{(i+n)}$ and $\Sigma \mathrm{DEHPm}$ showed no significant associations $(r=0.06-0.20, \quad P=0.173-0.490)$, but $\Sigma$ DiNPm showed a positive association approaching statistical significance $(r=0.17, P=0.058)$.

No significant correlations were found between phthalate concentrations and adolescent BMI, analysed as absolute values or as age- and gender-adjusted $Z$-scores and percentiles $(r=-0.10$ to 0.04 , $P=0.345-0.931)$.

There were no significant associations of ovarian volume and antral follicle count with antenatal phthalate metabolite exposure. There was no association of the phthalate MEP $(r=-0.15, P=0.101)$ and MEHP $(r=-0.04, P=0.642)$ with ovarian volume; further, there was no association of MiBP, MnBP, MBzP,

Table 2 Serum phthalate metabolite concentrations ( $\mathrm{ng} / \mathrm{ml})$ in 123 antenatal maternal serum samples of Australian mothers collected 1990-1991 (given as minimum, maximum and percentiles).

\begin{tabular}{|c|c|c|c|c|c|c|c|c|}
\hline $\mathrm{ng} / \mathrm{ml}$ & LOD* & $\boldsymbol{n}<$ LOD & Min & 25 & 50 & 75 & 90 & Max \\
\hline MEP & 0.92 & 18 & $<\mathrm{LOD}$ & 1.32 & 4.59 & 8.26 & 15.85 & 37.19 \\
\hline MiBP & 0.82 & 19 & $<\mathrm{LOD}$ & 1.13 & 1.77 & 2.85 & 6.16 & 34.52 \\
\hline $\mathrm{MnBP}$ & 0.77 & 2 & $<\mathrm{LOD}$ & 1.63 & 2.46 & 4.30 & 10.99 & 50.95 \\
\hline $\mathrm{MBzP}$ & 0.40 & 7 & $<\mathrm{LOD}$ & 0.79 & 1.26 & 2.11 & 3.87 & 24.31 \\
\hline MEHP & 0.60 & 46 & $<\mathrm{LOD}$ & $<$ LOD & 1.18 & 3.10 & 5.76 & 21.65 \\
\hline МЕСРP & 0.28 & 0 & 0.52 & 1.13 & 1.64 & 2.40 & 3.16 & 16.50 \\
\hline MiNP & 0.20 & 114 & $<\mathrm{LOD}$ & $<$ LOD & $<$ LOD & $<\mathrm{LOD}$ & $<\mathrm{LOD}$ & 1.22 \\
\hline $\mathrm{MCiOP}$ & 0.10 & 32 & $<$ LOD & $<\mathrm{LOD}$ & 0.17 & 0.34 & 0.59 & 2.03 \\
\hline$\Sigma \mathrm{MBP}_{(i+n)}$ & & 0 & 1.18 & 2.84 & 4.42 & 7.73 & 18.26 & 69.00 \\
\hline$\Sigma$ DEHPmetab & & 0 & 1.30 & 2.73 & 4.34 & 7.05 & 10.82 & 51.34 \\
\hline$\Sigma$ DiNPmetab & & 0 & 0.29 & 0.29 & 0.44 & 0.71 & 1.13 & 2.84 \\
\hline इall phth.m & & 0 & 6.64 & 13.54 & 19.12 & 29.54 & 53.39 & 114.41 \\
\hline
\end{tabular}

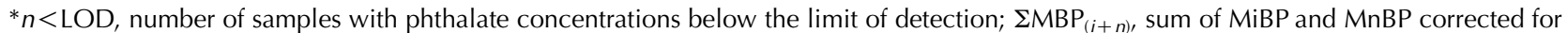
molecular weight; $\Sigma$ DEHPmetab, sum of all DEHP metabolites corrected for molecular weight; $\Sigma$ DiNPmetab, sum of all DiNP metabolites corrected for molecular weight; $\Sigma$ all phth.m, molar sum of all phthalate metabolites expressed as MEHP. 
Table 3 Correlations between quartiles of antenatal maternal serum phthalate metabolite (ng/ml) concentrations.

\begin{tabular}{|c|c|c|c|c|c|c|c|c|}
\hline & & & & $\Sigma$ DiNPm & इDEHPm & $\boldsymbol{\Sigma M B P}_{(i+n)}$ & & \\
\hline \multirow[t]{2}{*}{ MiNP } & $r$ & -0.010 & & 0.073 & $0.374^{+}$ & $0.698^{+}$ & $r$ & sall phth.m \\
\hline & $P$ & 0.910 & & 0.425 & 0.000 & 0.000 & $P$ & \\
\hline \multirow[t]{2}{*}{ MECPP } & $r$ & $0.518^{\dagger}$ & 0.107 & & $0.210^{*}$ & 0.069 & $r$ & $\Sigma$ DiNPm \\
\hline & $P$ & 0.000 & 0.239 & & 0.020 & 0.447 & $P$ & \\
\hline \multirow[t]{2}{*}{ MEHP } & $r$ & $-0.193^{*}$ & $0.200^{*}$ & 0.098 & & $0.199 *$ & $r$ & $\Sigma \mathrm{DEHPm}$ \\
\hline & $P$ & 0.032 & 0.027 & 0.279 & & 0.028 & $P$ & \\
\hline \multirow[t]{2}{*}{$\mathrm{MBzP}$} & $r$ & -0.042 & 0.134 & 0.097 & $0.359^{\dagger}$ & & & \\
\hline & $P$ & 0.643 & 0.140 & 0.286 & 0.000 & & & \\
\hline \multirow[t]{2}{*}{ MnBP } & $r$ & -0.007 & $0.322^{+}$ & 0.012 & 0.121 & $0.423^{+}$ & & \\
\hline & $P$ & 0.937 & 0.000 & 0.897 & 0.181 & 0.000 & & \\
\hline \multirow[t]{2}{*}{ MiBP } & $r$ & -0.068 & $0.183^{*}$ & 0.029 & $0.223^{*}$ & $0.290^{*}$ & $0.759^{\dagger}$ & \\
\hline & $P$ & 0.452 & 0.043 & 0.752 & 0.013 & 0.001 & 0.000 & \\
\hline \multirow[t]{3}{*}{ MEP } & $r$ & -0.032 & 0.068 & -0.101 & -0.017 & -0.002 & 0.026 & 0.085 \\
\hline & $P$ & 0.727 & 0.453 & 0.264 & 0.852 & 0.980 & 0.779 & 0.349 \\
\hline & & MCiOP & MiNP & МЕСРP & MEHP & $\mathrm{MBzP}$ & MnBP & MiBP \\
\hline
\end{tabular}

$r$, correlation; $P, P$ value, ${ }^{*} P<0.05$ and ${ }^{\dagger} P<0.01$. $\Sigma$ DiNPm, sum of all DiNP metabolites corrected for molecular weight; $\Sigma$ DEHPm, sum of all DEHP metabolites corrected for molecular weight; $\Sigma \mathrm{MBP}_{(i+n)}$, sum of MiBP and MnBP corrected for molecular weight; $\Sigma$ all phth.m, molar sum of all phthalate metabolites expressed as MEHP.

MECPP and MCiOP with ovarian volume $(r=0.05-0.10$, $P=0.291-0.575)$. With respect to antral follicle count, MEP, MBzP and MEHP $(r=-0.01$ to -011 , $P=0.252-0.887)$ and MiBP, MnBP, MECPP and MCiOP $(r=0.02-0.12, P=0.198-0.865)$ showed no correlation with antral follicle number.

Significantly higher serum concentrations of MEP and sall phthalate metabolites, but no other individual metabolites, were negatively associated with adolescent features of PCOS defined by the Rotterdam criteria ( $P=0.001$ and $P=0.005$ respectively; Fig. 1). Higher serum concentrations of MEP were also significantly negatively associated with adolescent PCO $(P=0.022$; Fig. 2). There were no significant associations between phthalate metabolites and adolescent PCOS as defined by NIH criteria (data not shown).

MEP, but no other metabolite, was significantly negatively associated with serum $\mathrm{AMH}$ concentrations ( $r=-0.21, P=0.031)$. MEHP, but no other metabolite, was significantly negatively associated with serum $\mathrm{FSH}$ ( $r=-0.22, P=0.024)$. No metabolite was significantly associated with serum levels of inhibin B.

\section{Maternal androgens and SHBG at 18 and 36 weeks of gestation}

SHBG concentrations at 18 weeks were significantly negatively correlated with MEHP $(r=-0.22, P=0.016)$ and with adjustment for maternal BMI at the time ( $r=-0.23, P=0.018)$. The correlation between SHBG and MEP was approaching statistical significance ( $r=-0.17, P=0.072$; Table 4 ) and with adjustment for maternal BMI $(r=-0.16, P=0.94)$. $\Sigma$ DiNPm was significantly negatively associated with maternal concentrations of androstenedione $(r=-0.19$, $P=0.035)$ and DHEAS $(r=-0.24, P=0.008)$.

SHBG levels at 36 weeks were significantly negatively correlated with maternal serum MEHP concentrations
( $r=-0.23, P=0.016)$ and on controlling for current maternal BMI $(r=-0.24, P=0.010)$ and positively correlated with MECPP without and with adjustment for maternal BMI $(r=0.24, P=0.009$, and $r=0.24$, $P=0.010$ respectively). The positive correlation of SHBG and MCiOP concentrations approached statistical significance univariately $(r=0.17, P=0.077$; Table 5) and after controlling for maternal BMI $(r=0.16$, $P=0.085)$. There were no significant associations between maternal serum phthalate levels and maternal concentrations of androgens.



Figure 1 Association between the sum of all phthalate metabolites ( all phthalate metabolites adjusted for molecular weight) in antenatal maternal serum samples and polycystic ovarian syndrome in adolescent daughters as defined by the Rotterdam criteria. Medians, IQRs (box plots) together with the whisker that extend to the minimum and maximum values in the data range inside 1.5 times the interquartile range (box length) from the upper and lower ends of the box. Outliers and extreme values are shown with 'open circle' and ${ }^{\prime * \prime}$ respectively. 


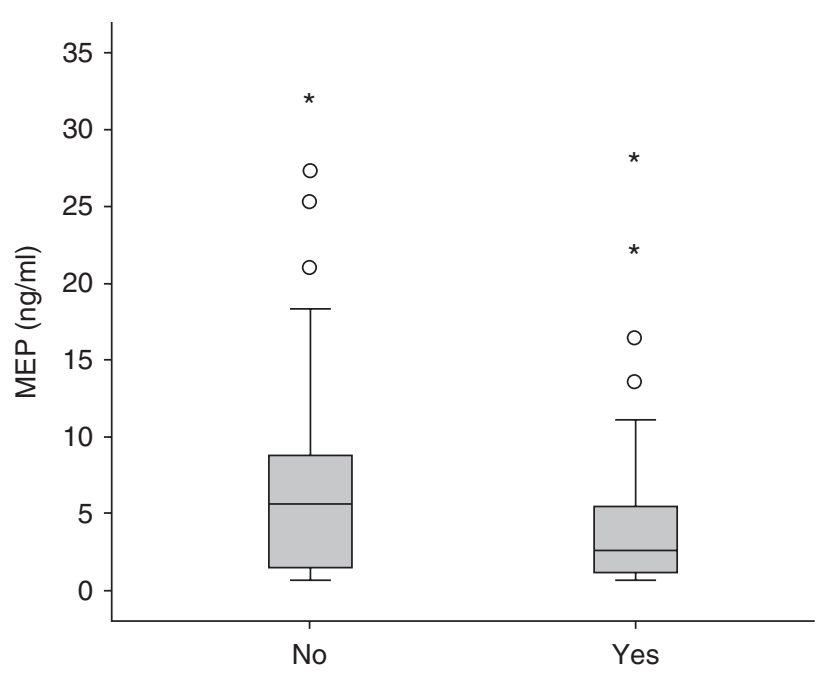

Figure 2 Association between the concentration of the phthalate metabolite MEP in antenatal maternal serum and ultrasounddetermined PCO in their adolescent daughters. Medians, IQRs (box plots) together with the whisker that extend to the minimum and maximum values in the data range inside 1.5 times the interquartile range (box length) from the upper and lower ends of the box. Outliers and extreme values are shown with 'open circle' and '*' respectively.

\section{Discussion}

Using prenatal serum samples from a large and unique prospective cohort study of pregnant women and their adolescent daughters, we report for the first time that antenatal exposure to phthalates may be associated with long-term effects on reproductive development, including a significantly increased uterine volume and potentially a protective effect of phthalate exposure against $\mathrm{PCO}$ in girls. Together with our finding of a negative association between MEP levels in maternal serum during pregnancy, and serum $\mathrm{AMH}$ and antral follicle count in their daughters at 15 years of age, the data suggest that fetal exposure to phthalates may influence ovarian follicle number in adolescence. We hypothesise that the mechanism behind the effects seen in girls exposed to higher levels of phthalates may be impaired steroidogenesis resulting in altered fetal androgen synthesis. Owing to the multiple comparisons performed, we cannot exclude the possibility that any associations determined arose by chance.

Phthalates are present within cosmetics, plastics, detergents, solvents and lubricants, clothing and food packaging (phthalate diesters can also enter serum samples through contamination from plastic surfaces during blood sampling, processing, storage and analysis). Consequently, it is virtually impossible to avoid chronic low-level exposure to phthalates. It is of note that MEHP, a metabolite of DEHP, which has been reported to lead to oocyte atresia (Takai et al. 2009), suppresses oestradiol $\left(\mathrm{E}_{2}\right)$ synthesis and ovulation in female rats. In addition, MEHP leads to a reduced size of pre-ovulatory follicles and suppresses follicular development when cultured in vitro (Wang et al. 2012). The mechanism of action is unknown, but may be via inhibition of ovarian steroidogenesis or a consequence of oxidative damage (Wang et al. 2012). The increased uterine volume may be due to an oestrogenic influence, antenatally or during childhood, as uterine volume is a marker of oestrogenic exposure (Lethaby \& Vollenhoven 2008).

The link between higher prenatal exposures to phthalates and lower AMH levels in adolescence may theoretically be explained by a negative effect of phthalate exposure on granulosa cell growth and function, or at least AMH secretion from granulosa cells. This interpretation is consistent with studies performed on rats, where histological analysis of preovulatory follicles demonstrated a reduction in size of DEHP-exposed granulosa cells (Davis et al. 1994). Interestingly, in a previous study, we found that circulating maternal testosterone concentrations at 18 weeks of gestation predicted $\mathrm{AMH}$ in adolescence (Hart et al. 2010a, 2010b).

We have recently shown that $\mathrm{AMH}$ levels in childhood and adolescence are highly stable and appear to accurately reflect the individual follicle pool (Hagen et al. 2012) as well as ovarian reserve (Hagen et al. 2010). While our present finding that antenatal exposure to phthalates is associated with a suppression of circulating serum FSH in adolescence would appear contradictory, we cannot exclude a potential pituitary programming effect due to a resetting of the hypothalamic-pituitary-ovarian axis following phthalate exposure. Whether our findings suggest that phthalate exposure will lead to a reduction in ovarian reserve and potentially reduced reproductive capacity will only be determined with further follow-up.

While this pilot study was performed in a subset of the Raine cohort, we believe the sample was representative of the wider Raine cohort as the median age at menarche was 12.4 years, consistent with recently published studies in similar populations (Sloboda et al. 2011). The girls recruited to this study had a slightly younger age at menarche compared with the non-recruited girls from the entire Raine cohort (median ages 12.4 and 13 respectively). However, due to the timing of the assessments at the commencement of a menstrual cycle at the end of a school day, recruitment was challenging, and the mothers and daughters were unaware of this study endpoint; therefore, the chances of recruitment bias were expected to be slim.

In rats, adverse effects of phthalate exposure on male reproduction have been demonstrated for DBP, DEHP and MEHP, especially when prenatal exposure occurred during a sensitive developmental window early in pregnancy (Sharpe \& Skakkebaek 2008), or exposure was both pre- and post-natal (Jobling et al. 2011). 
Table 4 Correlation between quartiles of antenatal maternal serum phthalate metabolite concentration and log-transformed maternal androgens at 18 weeks $(n=119)$.

\begin{tabular}{|c|c|c|c|c|c|c|}
\hline $\begin{array}{l}\text { Phthalate } \\
\text { metabolites (ng/ml) }\end{array}$ & A4 (nmol/l) & DHEAS $(\mu \mathrm{mol} / \mathrm{l})$ & TT $(\mathrm{pmol} / \mathrm{l})$ & SHBG $(\mathrm{nmol} / \mathrm{l})$ & cFT (pmol/l) & FAI \\
\hline MEP & 0.109 & 0.058 & 0.094 & $-0.165^{*}$ & 0.151 & $0.157^{*}$ \\
\hline MiBP & 0.023 & -0.042 & 0.003 & 0.108 & -0.061 & -0.051 \\
\hline MnBP & -0.030 & -0.112 & -0.022 & 0.048 & -0.053 & -0.041 \\
\hline MBzP & -0.006 & -0.057 & -0.009 & -0.123 & 0.037 & 0.053 \\
\hline MEHP & 0.090 & $0.164 *$ & 0.007 & $-0.216^{\dagger}$ & 0.119 & 0.111 \\
\hline МЕСРP & -0.018 & -0.075 & -0.001 & $0.171^{*}$ & -0.068 & -0.084 \\
\hline MCiOP & -0.149 & $-0.185^{\dagger}$ & -0.050 & $0.165^{*}$ & -0.105 & -0.121 \\
\hline$\Sigma \mathrm{MBP}_{(i+n)}$ & -0.052 & -0.081 & -0.033 & 0.043 & -0.061 & -0.048 \\
\hline$\Sigma \mathrm{DEHP}$ & 0.122 & 0.134 & -0.015 & -0.036 & 0.016 & 0.005 \\
\hline$\Sigma$ DiNP & $0.193^{+}$ & $0.242^{\ddagger}$ & -0.064 & 0.135 & -0.103 & -0.118 \\
\hline इall phth.m & 0.086 & 0.032 & 0.047 & -0.097 & 0.071 & 0.085 \\
\hline
\end{tabular}

*P=0.05-0.1, ${ }^{\dagger} P<0.05$ and ${ }^{\ddagger} P<0.01 . \Sigma \mathrm{MBP}_{(i+n)}$, sum of MiBP and MnBP corrected for molecular weight; $\Sigma$ DiNPm, sum of all DiNP metabolites corrected for molecular weight; $\Sigma$ DEHPm, sum of all DEHP metabolites corrected for molecular weight; $\Sigma$ all phth.m, molar sum of all phthalate metabolites expressed as MEHP.

Recently, it has been questioned whether phthalates have similar effects in the human fetal testis (Welsh et al. 2008, Mitchell et al. 2012). There are several known mechanisms of action for these effects on the male rat, such as the inhibition of testosterone synthesis (Auharek et al. 2010, Christiansen et al. 2010, MacLeod et al. 2010, Boberg et al. 2011), inhibition of $E_{2}$ synthesis in granulosa cells (Reinsberg et al. 2009) as well as inhibition of aromatase activity (van Meeuwen et al. 2008), prostaglandin synthesis (Kristensen et al. 2011) and possibly effects via inhibition of the thyroid axis (Meeker \& Ferguson 2011). This makes the interpretation of in vivo effects in humans very complex. It is, however, plausible that phthalate exposure may alter the subtle testosterone-oestrogen balance that is needed to provide the optimal endocrine environment for development and function of the reproductive organs in both sexes. Furthermore, it is believed that the dose-response profiles of endocrine disrupters are not uniform, as in some instances even very low levels of chemicals may have an endocrine disrupting effect, making interpretation of the data very complex (Fagin 2012).
A potentially significant limitation of our study relates to the age and storage conditions of our maternal serum samples. It is known that the enzymes involved in hydrolysis of diester phthalates to monoester phthalates are present in blood. They may be responsible for diester to monoester conversion after blood samples are drawn (Hines et al. 2009), and the speed of this conversion is dependent on the length of the alkyl chain, with shorter alkyl chains undergoing rapid hydrolisation and longer alkyl chains, such as DEHP, taking significantly longer (Kato et al. 2003). For that reason, analysis of monoester phthalates may yield falsely elevated levels due to ex vivo conversion of diester contamination during blood sampling, storage and handling in the laboratory. Some second-step metabolites are also found in serum, albeit at lower concentrations than in urine. Thus, to inhibit enzyme activity, best practice requires that acid should be added to blood samples immediately after collection and centrifugation for serum isolation. As samples in this study were collected 20 years ago and were stored without acid addition, we performed a series of pilot studies to ensure that the results of the phthalate

Table 5 Correlation between quartiles of antenatal maternal serum phthalate metabolite concentration and log-transformed maternal androgens at 36 weeks of gestation $(n=114)$.

\begin{tabular}{|c|c|c|c|c|c|c|}
\hline $\begin{array}{l}\text { Phthalate } \\
\text { metabolites }(\mathrm{ng} / \mathrm{ml})\end{array}$ & A4 $(\mathrm{nmol} / \mathrm{l})$ & DHEAS $(\mu \mathrm{mol} / \mathrm{l})$ & TT $(\mathrm{pmol} / \mathrm{l})$ & SHBG (nmol/l) & cFT (pmol/l) & FAI \\
\hline MEP & 0.115 & 0.048 & 0.071 & -0.024 & 0.068 & 0.065 \\
\hline MiBP & -0.060 & -0.084 & -0.101 & -0.020 & -0.063 & -0.064 \\
\hline $\mathrm{MnBP}$ & -0.035 & -0.058 & -0.052 & -0.101 & 0.010 & 0.016 \\
\hline $\mathrm{MBzP}$ & -0.045 & -0.132 & -0.063 & -0.149 & 0.027 & 0.033 \\
\hline MEHP & $0.179 *$ & 0.045 & 0.017 & $-0.226^{\dagger}$ & 0.127 & 0.133 \\
\hline МЕСРP & 0.055 & -0.055 & 0.065 & $0.244^{\ddagger}$ & -0.079 & -0.082 \\
\hline MCiOP & -0.094 & -0.057 & -0.005 & $0.166^{*}$ & -0.092 & -0.092 \\
\hline$\Sigma \mathrm{MBP}_{(i+n)}$ & -0.050 & -0.007 & -0.053 & -0.102 & 0.014 & 0.015 \\
\hline$\Sigma$ DEHP & $0.184^{*}$ & 0.077 & 0.011 & -0.034 & 0.023 & 0.026 \\
\hline$\Sigma$ DiNP & -0.087 & -0.120 & 0.019 & 0.097 & -0.039 & -0.037 \\
\hline इall phth.m & 0.136 & 0.059 & 0.061 & -0.089 & 0.094 & 0.093 \\
\hline
\end{tabular}

${ }^{*} P=0.05-0.1,{ }^{\dagger} P<0.05$ and ${ }^{\ddagger} P<0.01$. TT, total testosterone - measured with ultrasensitive assay; FAl, free testosterone index; $\Sigma \mathrm{MBP}(i+n)$, sum of MiBP and MnBP corrected for molecular weight; $\Sigma$ DiNPm, sum of all DiNP metabolites corrected for molecular weight; $\Sigma$ DEHPm, sum of all DEHP metabolites corrected for molecular weight; $\Sigma$ all phth.m, molar sum of all phthalate metabolites expressed as MEHP. 
analysis were robust and likely to reflect true phthalate exposure at the time of collection. We analysed monoesters (first-step metabolites) and second-step metabolites for DEHP and DiNP, which arise from hepatic and renal metabolism of endogenous sources. It is believed that second-step metabolites are a good biomarker of true exposure as they only occur after passage through the body, i.e. cannot be caused through contamination. Diester contamination, in particular to DEHP and DBP, and conversion after/during the drawing of blood, centrifugation, aliquoting and storage might well have occurred. However, this is likely to be relatively minor, as we observed generally very low levels of first-step metabolites in ranges that have been reported before. In addition, there was a large interindividual variation for all the metabolites instead of a generally high, systemic concentration in all samples. Many researchers in this field advocate the use of urine samples for the assessment of phthalate exposure in humans, as these contain both first- and second-step metabolites of phthalate diesters; unfortunately, this was not available for this cohort. However, urinary phthalate concentrations can also vary considerably due to differences, other than exposure differences, such as urinary dilution, kidney function and the metabolic changes during pregnancy and developmental maturation of metabolic detoxification pathways.

It is also important to note that the serum levels of phthalate metabolites this study are in accordance with those observed in other studies. For instance, the median levels in serum from USA adults (NHANES 1999-2000) were reported to be $4.1 \mathrm{ng} / \mathrm{ml}$ (MEP), $14.4 \mathrm{ng} / \mathrm{ml}$ (MBP) and $5.4 \mathrm{ng} / \mathrm{ml}$ (MEHP) (Silva et al. 2004). A recent study of an elderly population in Sweden showed levels in the similar range $11.6 \mathrm{ng} / \mathrm{ml}$ (MEP), $13.5 \mathrm{ng} / \mathrm{ml}$ (MiBP) and $4.53 \mathrm{ng} / \mathrm{ml}$ (MEHP) (Olsen et al. 2012). In a study of young Danish men, medium levels of MEP, MiBP and MnBP were found to be below detection limits, but median MEHP serum concentrations were reported as $7.88 \mathrm{ng} / \mathrm{ml}$ (Frederiksen et al. 2010). Therefore, we believe that despite the potential collection and storage limitations, the phthalate levels measured in this study are likely to accurately reflect in vivo exposure at the time of collection. Based on these considerations, we have adapted a conservative approach and analysed the monoester phthalate results as semiquantitative data. This approach means that the absolute value reflects in a way the 'order of magnitude' that this metabolite was detected within the sample. By contrast, the carboxylated metabolites of DEHP and DiNP are secondary metabolites, and, therefore, their levels will not be influenced by variable activity of serum enzymes during the long storage period. These results were, therefore, accepted as quantitative data.

Our finding that MEP concentrations are associated with several reproductive outcomes in adolescent girls has, to date, not been replicated in rodent studies.
On the one hand, this may reflect a potential species difference. Precedence for this exists in human studies on male reproductive effects, which also found significant associations with MEP (Swan et al. 2005, Main et al. 2006b). Alternatively, as the sources of MEP exposure are predominantly personal care products, MEP levels may be a surrogate for the presence of other hormone disrupting chemicals within these products (e.g. sun screens or parabens). As we did not measure these other chemicals, we cannot exclude or confirm such a confounding effect.

It is important to point out that any associations derived from these analyses of antenatal phthalate exposure cannot confirm a direct causal relationship with outcome. In addition, we have no data on the nature and magnitude of the post-natal environmental exposure of the teenage girls, which may contribute to our findings. Humans are exposed to many phthalates simultaneously, along with other environmental chemicals that may also exert dose-dependent, endocrine disrupting effects (Kortenkamp 2007).

\section{Conclusion}

This pilot study suggests that maternal exposure to environmental phthalates during pregnancy may be associated with long-term oestrogenic, or anti-androgenic, effects on the reproductive development of their daughters, including a significantly increased uterine volume. It also appeared that maternal exposure to phthalates may protect against PCO in their offspring. However, as serum samples had been stored for more than two decades before analysis, our findings need to be corroborated in further prospective cohort studies.

\section{Discussion from meeting}

Jerry Heindel (NIEHS, USA): What are the levels and ranges of prenatal exposure to the different phthalates? If there is not a sufficient difference between the high and the low levels, it will be difficult to assess their effects.

R Hart (Perth, Australia): The phthalate levels were consistent with the NHANES published data with no surprisingly high values and very few outliers.

K M Main (Copenhagen, Denmark): We did not know what to expect concerning the levels of phthalates in pregnant women 20 years ago in Australia and whether they would be similar to the situation in the UK and USA. Australia was found to be similar to other countries including a vast range between the highest and lowest levels. Some women were enormously exposed, far above the median.

Hagai Levine (Jerusalem, Israel): Did you look at other effects of prenatal exposure on pubertal development such as Tanner stage or breast development?

R Hart: We had planned to look at these, but the girls were unwilling to be staged by nurses. We relied on 
self-staging, but this has been shown to be unreliable and not robust to scrutiny. Therefore, we did not analyse these parameters.

Germaine Buck Louis (Rockville, USA): Was there an association between phthalate exposure and BMI over time prior to the outcomes you described? Inclusion of BMI into your model could possibly identify this parameter as an intermediate.

R Hart: We were expecting an association between phthalates and BMI, but in our analysis, there was no correlation in any of the phthalates examined.

Sheela Sathyanarayana (Seattle, USA): Why did you pool the blood samples from 18 and 34 weeks of gestation? These are different developmental time periods for urogenital development and might have been associated with different effects.

R Hart: We considered analysing both samples but the stored material is a valuable but scarce resource. With the agreement of the committee, we pooled half of each sample. Phthalate levels are short lived after exposure and a single-day sample might not be representative of the long-term levels. We assume that the lifestyle did not alter significantly between 18 and 34 weeks and that pooled samples would be more representative of levels across gestation.

\section{Declaration of interest}

D Sloboda, M Hickey, J A Keelan, J P Newnham, D A Doherty and C E Pennell declare that they have no relationship with the meeting sponsors. R Hart received sponsorship from the organising committee to attend the Copenhagen Workshop on Endocrine Disruptors 2013. N E Skakkebæk, H Frederiksen and K M Main have received funding from the Danish Ministry of the Environment - Environmental Protection Agency for other research not included in this paper. K M Main has also been a member of an advisory group related to human studies on pesticide effects.

\section{Funding}

The Australian group was supported by a NHMRC project grant (number 403968) and by a University of Western Australia Ada Bartholomew grant. M Hickey is funded by an NHMRC Clinical Career Development Award. The Danish research team was supported by a grant from The Danish Agency for Science, Technology and Innovation (09-067180) and the Velux Foundation. This article is based on work presented at the 7th Copenhagen Workshop on Endocrine Disrupters, which was supported by the Danish Ministry of the Environment Environmental Protection Agency. Publication of this special issue was supported by the Society for Reproduction and Fertility. The collection of maternal data and samples was funded by NH\&MRC, The Raine Medical Research Foundation and The Women and Infants' Research Foundation (WIRF) while the collection of adolescent data and samples was funded by NHMRC project grant number 403968 and by a UWA Ada Bartholomew grant. M Hickey was funded by an
NHMRC Clinical Career Development Award. The Danish research team was supported by a grant from The Danish Agency for Science, Technology and Innovation (09-067180) and the Velux Foundation.

\section{Author contribution statement}

$\mathrm{R}$ Hart initiated the project, facilitated the scientific collaboration and was chiefly responsible for writing the manuscript. D A Doherty performed the data analysis and assisted with manuscript preparation. $\mathrm{H}$ Frederiksen performed the phthalate analysis and assisted with manuscript preparation. J A Keelan, M Hickey, D Sloboda, C E Pennell, J P Newnham, and N E Skakkebaek provided scientific input to the study and assisted with manuscript preparation. K M Main provided scientific input to the study, coordinated phthalate analysis and took a lead role with manuscript preparation.

\section{Acknowledgements}

The authors are extremely grateful to The Raine Study participants and their families who took part in this study and the Raine Study Team for cohort co-ordination and data collection, the NH\&MRC for their long-term contribution to funding the study over the last 20 years and the Telethon Institute for Child Health Research for long-term support of the Study. They acknowledge the Raine Medical Research Foundation, The Telethon Institute of Child Health Research, The University of Western Australia, UWA Faculty of Medicine, Dentistry and Health Sciences, Women's and Infant's Research Foundation and Curtin University for financial support and for providing funding for core management of the Raine Study.

\section{References}

Abbott DH, Barnett DK, Bruns CM \& Dumesic DA 2005 Androgen excess fetal programming of female reproduction: a developmental aetiology for polycystic ovary syndrome? Human Reproduction Update 11 357-374. (doi:10.1093/humupd/dmi013)

Adams J, Franks S, Polson DW, Mason HD, Abdulwahid N, Tucker M, Morris DV, Price J \& Jacobs HS 1985 Multifollicular ovaries: clinical and endocrine features and response to pulsatile gonadotropin releasing hormone. Lancet 2 1375-1379. (doi:10.1016/S0140-6736(85)92552-8)

Adibi JJ, Whyatt RM, Hauser R, Bhat HK, Davis BJ, Calafat AM, Hoepner LA, Perera FP, Tang D \& Williams PL 2010 Transcriptional biomarkers of steroidogenesis and trophoblast differentiation in the placenta in relation to prenatal phthalate exposure. Environmental Health Perspectives 118 291-296. (doi:10.1289/ehp.0900788)

Andersson AM, Jorgensen N, Main KM, Toppari J, Rajpert-De Meyts E, Leffers H, Juul A, Jensen TK \& Skakkebaek NE 2008 Adverse trends in male reproductive health: we may have reached a crucial 'tipping point'. International Journal of Andrology 31 74-80. (doi:10.1111/j.1365-2605. 2007.00853.x)

Auharek SA, de Franca LR, McKinnell C, Jobling MS, Scott HM \& Sharpe RM 2010 Prenatal plus postnatal exposure to di(n-butyl) phthalate and/or flutamide markedly Definitions. Inreduces Sertoli cell number in the rat. Endocrinology and Metabolism Clinics of North America 15 2868-2875.

Balen AH, Laven JSE, Tan S-L \& Dewailly D 2003 The ultrasound assessment of the polycystic ovary: International Consensus Definitions. In ASRM/ESHRE Consensus Meeting on Polycystic Ovary Syndrome, pp 1-25. 
Boberg J, Christiansen S, Axelstad M, Kledal TS, Vinggaard AM, Dalgaard M, Nellemann C \& Hass U 2011 Reproductive and behavioral effects of diisononyl phthtalate (DINP) in perinatally exposed rats. Reproductive Toxicology 31 200-209. (doi:10.1016/j.reprotox.2010. 11.001)

Christiansen S, Boberg J, Axelstad M, Dalgaard M, Vinggaard AM, Metzdorff SB \& Hass U 2010 Low-dose perinatal exposure to di(2-ethylhexyl) phthalate induces anti-androgenic effects in male rats. Reproductive Toxicology 30 313-321. (doi:10.1016/j.reprotox.2010.04. 005)

Cole TJ, Bellizzi MC, Flegal KM \& Dietz WH 2000 Establishing a standard definition for child overweight and obesity worldwide: international survey. British Medical Journal 320 (7244) 1240-1243.

Davis BJ, Maronpot RR \& Heindel JJ 1994 Di-(2-ethylhexyl) phthalate suppresses estradiol and ovulation in cycling rats. Toxicology and Applied Pharmacology 128 216-223. (doi:10.1006/taap.1994.1200)

Desdoits-Lethimonier C, Albert O, Le Bizec B, Perdu E, Zalko D, Courant F, Lesne L, Guille F, Dejucq-Rainsford N \& Jegou B 2012 Human testis steroidogenesis is inhibited by phthalates. Human Reproduction $\mathbf{2 7}$ 1451-1459. (doi:10.1093/humrep/des069)

ESHRE/ASRM Rotterdam Consensus Meeting 2004a Revised 2003 consensus on diagnostic criteria and long-term health risks related to polycystic ovary syndrome (PCOS). Human Reproduction 19 41-47. (doi:10.1093/humrep/deh098)

ESHRE/ASRM 2004b Revised 2003 consensus on diagnostic criteria and long-term health risks related to polycystic ovary syndrome. Fertility and Sterility 81 19-25.

Fagin D 2012 Toxicology: the learning curve. Nature 490 462-465. (doi:10.1038/490462a)

Frederiksen H, Skakkebaek NE \& Andersson AM 2007 Metabolism of phthalates in humans. Molecular Nutrition \& Food Research 51 899-911. (doi:10.1002/mnfr.200600243)

Frederiksen H, Jorgensen N \& Andersson AM 2010 Correlations between phthalate metabolites in urine, serum, and seminal plasma from young Danish men determined by isotope dilution liquid chromatography tandem mass spectrometry. Journal of Analytical Toxicology 34 400-410. (doi:10.1093/jat/34.7.400)

Frederiksen H, Aksglaede L, Sorensen K, Skakkebaek NE, Juul A \& Andersson AM 2011 Urinary excretion of phthalate metabolites in 129 healthy Danish children and adolescents: estimation of daily phthalate intake. Environmental Research 111 656-663. (doi:10.1016/j.envres. 2011.03.005)

Frederiksen $H$, Sorensen $K$, Mouritsen A, Aksglaede L, Hagen CP, Petersen JH, Skakkebaek NE, Andersson AM \& Juul A 2012 High urinary phthalate concentration associated with delayed pubarche in girls. International Journal of Andrology 35 216-226. (doi:10.1111/ j.1365-2605.2012.01260.x)

Hagen CP, Aksglaede L, Sorensen K, Main KM, Boas M, Cleemann L, Holm K, Gravholt CH, Andersson AM, Pedersen AT et al. 2010 Serum levels of anti-Müllerian hormone as a marker of ovarian function in 926 healthy females from birth to adulthood and in 172 Turner syndrome patients. Journal of Clinical Endocrinology and Metabolism 95 5003-5010. (doi:10.1210/jc.2010-0930)

Hagen CP, Aksglaede L, Sorensen K, Mouritsen A, Andersson AM, Petersen JH, Main KM \& Juul A 2012 Individual serum levels of antiMüllerian hormone in healthy girls persist through childhood and adolescence: a longitudinal cohort study. Human Reproduction $\mathbf{2 7}$ 861-866. (doi:10.1093/humrep/der435)

Handelsman DJ 2001 Estrogens and falling sperm counts. Reproduction, Fertility, and Development 13 317-324. (doi:10.1071/RD00103)

Hart R, Sloboda DM, Doherty DA, Norman RJ, Atkinson HC, Newnham JP, Dickinson JE \& Hickey M 2009 Prenatal determinants of uterine volume and ovarian reserve in adolescence. Journal of Clinical Endocrinology and Metabolism 94 4931-4937. (doi:10.1210/jc.2009-1342)

Hart R, Doherty DA, Norman RJ, Franks S, Dickinson JE, Hickey M \& Sloboda DM 2010a Serum antiMüllerian hormone (AMH) levels are elevated in adolescent girls with polycystic ovaries and the polycystic ovarian syndrome (PCOS). Fertility and Sterility 94 1118-1121. (doi:10.1016/j.fertnstert.2009.11.002)

Hart R, Sloboda D, Doherty D, Norman R, Atkinson H, Newnham J, Dickinson J \& Hickey M 2010 b Circulating maternal testosterone concentrations at 18 weeks of gestation predict circulating levels of
antiMüllerian hormone in adolescence: a prospective cohort study. Fertility and Sterility 94 1544-1547. (doi:10.1016/j.fertnstert.2009.12. 060)

Hickey M, Sloboda DM, Atkinson HC, Doherty DA, Franks S, Norman RJ, Newnham JP \& Hart R 2009 The relationship between maternal and umbilical cord androgen levels and polycystic ovary syndrome in adolescence: a prospective cohort study. Journal of Clinical Endocrinology and Metabolism 94 3714-3720. (doi:10.1210/jc.2009-0544)

Hickey M, Doherty DA, Atkinson H, Sloboda DM, Franks S, Norman RJ \& Hart R 2011 Clinical, ultrasound and biochemical features of polycystic ovary syndrome in adolescents: implications for diagnosis. Human Reproduction 26 1469-1477.

Hines EP, Calafat AM, Silva MJ, Mendola P \& Fenton SE 2009 Concentrations of phthalate metabolites in milk, urine, saliva, and serum of lactating North Carolina women. Environmental Health Perspectives 117 86-92. (doi:10.1289/ehp.11610)

Hsu NY, Lee CC, Wang JY, Li YC, Chang HW, Chen CY, Bornehag CG, Wu PC, Sundell J \& Su HJ 2012 Predicted risk of childhood allergy, asthma, and reported symptoms using measured phthalate exposure in dust and urine. Indoor Air 22 186-199. (doi:10.1111/j.1600-0668.2011. 00753.x)

Huang PC, Kuo PL, Chou YY, Lin SJ \& Lee CC 2009 Association between prenatal exposure to phthalates and the health of newborns. Environment International 35 14-20. (doi:10.1016/j.envint.2008.05.012)

Janjua NR, Frederiksen H, Skakkebaek NE, Wulf HC \& Andersson AM 2008 Urinary excretion of phthalates and paraben after repeated whole-body topical application in humans. International Journal of Andrology 31 118-130. (doi:10.1111/j.1365-2605.2007.00841.x)

Jobling MS, Hutchison GR, van den Driesche S \& Sharpe RM 2011 Effects of di(n-butyl) phthalate exposure on foetal rat germ-cell number and differentiation: identification of age-specific windows of vulnerability. International Journal of Andrology 34 e386-e396. (doi:10.1111/j.13652605.2010.01140.x)

Jurewicz J \& Hanke W 2011 Exposure to phthalates: reproductive outcomes and children health. A review of epidemiological studies. International Journal of Occupational Medicine and Environmental Health 24 115-141. (doi:10.2478/s13382-011-0022-2)

Kato K, Silva MJ, Brock JW, Reidy JA, Malek NA, Hodge CC, Nakazawa H, Needham LL \& Barr DB 2003 Quantitative detection of nine phthalate metabolites in human serum using reversed-phase high-performance liquid chromatography-electrospray ionization-tandem mass spectrometry. Journal of Analytical Toxicology 27 284-289. (doi:10.1093/ jat/27.5.284)

Kortenkamp A 2007 Ten years of mixing cocktails: a review of combination effects of endocrine-disrupting chemicals. Environmental Health Perspectives 115 (Suppl 1) 98-105. (doi:10.1289/ehp.9357)

Kristensen D, Skalkam M, Audouze K, Lesne L, Desdoits-Lethimonier C, Frederiksen H, Brunak S, Skakkebaek N, Jegou B, Hansen J et al. 2011 Many putative endocrine disruptors inhibit prostaglandin synthesis. Environmental Health Perspectives 119 534-541. (doi:10.1289/ehp. 1002635)

Lethaby AE \& Vollenhoven BJ 2008 An evidence-based approach to hormonal therapies for premenopausal women with fibroids. Best Practice \& Research. Clinical Obstetrics \& Gynaecology 22 307-331. (doi:10.1016/j.bpobgyn.2007.07.010)

Lyche JL, Gutleb AC, Bergman A, Eriksen GS, Murk AJ, Ropstad E, Saunders M \& Skaare JU 2009 Reproductive and developmental toxicity of phthalates. Journal of Toxicology and Environmental Health. Part B, Critical Reviews 12 225-249. (doi:10.1080/10937400903094091)

Macdonald W, Newnham J, Gurrin L \& Evans S 1996 Effect of frequent prenatal ultrasound on birthweight: follow up at 1 year of age. Western Australian Pregnancy Cohort (Raine) Working Group. Lancet 348482. (doi:10.1016/S0140-6736(05)64587-4)

MacLeod DJ, Sharpe RM, Welsh M, Fisken M, Scott HM, Hutchinson GR, Drake AJ \& van den Driesche S 2010 Androgen action in the masculinization programming window and development of male reproductive organs. International Journal of Andrology 33 279-287. (doi:10.1111/j.1365-2605.2009.01005.x)

Main KM, Mortensen GK, Kaleva MM, Boisen KA, Damgaard IN, Chellakooty M, Schmidt IM, Suomi AM, Virtanen HE, Petersen DV et al. 2006a Human breast milk contamination with phthalates and 
alterations of endogenous reproductive hormones in infants three months of age. Environmental Health Perspectives 114 270-276. (doi:10.1289/ ehp.8075)

Main KM, Toppari J, Suomi AM, Kaleva M, Chellakooty M, Schmidt IM, Virtanen HE, Boisen KA, Kai CM, Damgaard IN et al. 2006 $b$ Larger testes and higher inhibin B levels in Finnish than in Danish newborn boys. Journal of Clinical Endocrinology and Metabolism 91 2732-2737. (doi:10.1210/jc.2005-2443)

Massart F, Parrino R, Seppia P, Federico G \& Saggese G 2006 How do environmental estrogen disruptors induce precocious puberty? Minerva Pediatrica 58 247-254.

Meeker JD \& Ferguson KK 2011 Relationship between urinary phthalate and bisphenol A concentrations and serum thyroid measures in U.S. adults and adolescents from the National Health and Nutrition Examination Survey (NHANES) 2007-2008. Environmental Health Perspectives 119 1396-1402. (doi:10.1289/ehp.1103582)

van Meeuwen JA, van Son O, Piersma AH, de Jong PC \& van den Berg M 2008 Aromatase inhibiting and combined estrogenic effects of parabens and estrogenic effects of other additives in cosmetics. Toxicology and Applied Pharmacology 230 372-382. (doi:10.1016/j.taap.2008.03.002)

Mitchell RT, Childs AJ, Anderson RA, van den Driesche S, Saunders PT, McKinnell C, Wallace WH, Kelnar CJ \& Sharpe RM 2012 Do phthalates affect steroidogenesis by the human fetal testis? Exposure of human fetal testis xenografts to di- $n$-butyl phthalate Journal of Clinical Endocrinology and Metabolism 97 E341-E348. (doi:10.1210/jc.2011-2411)

Mose T, Mortensen GK, Hedegaard M \& Knudsen LE 2007 Phthalate monoesters in perfusate from a dual placenta perfusion system, the placenta tissue and umbilical cord blood. Reproductive Toxicology 23 83-91. (doi:10.1016/j.reprotox.2006.08.006)

Olsen L, Lampa E, Birkholz DA, Lind L \& Lind PM 2012 Circulating levels of bisphenol A (BPA) and phthalates in an elderly population in Sweden, based on the Prospective Investigation of the Vasculature in Uppsala Seniors (PIVUS). Ecotoxicology and Environmental Safety 75 242-248. (doi:10.1016/j.ecoenv.2011.09.004)

Orsini LF, Venturoli S, Lorusso R, Pluchinotta V, Paradisi R \& Bovicelli L 1985 Ultrasonic findings in polycystic ovarian disease. Fertility and Sterility 43 709-714.

Pierik FH, Burdorf A, Deddens JA, Juttmann RE \& Weber RF 2004 Maternal and paternal risk factors for cryptorchidism and hypospadias: a casecontrol study in newborn boys. Environmental Health Perspectives 112 1570-1576. (doi:10.1289/ehp.7243)

Reinsberg J, Wegener-Toper P, van der Ven K, van der Ven $\mathbf{H}$ \& Klingmueller D 2009 Effect of mono-(2-ethylhexyl) phthalate on steroid production of human granulosa cells. Toxicology and Applied Pharmacology 239 116-123. (doi:10.1016/j.taap.2009.05.022)

Sharpe RM \& Skakkebaek NE 2008 Testicular dysgenesis syndrome: mechanistic insights and potential new downstream effects. Fertility and Sterility 89 e33-e38. (doi:10.1016/j.fertnstert.2007.12.026)

Silva MJ, Barr DB, Reidy JA, Malek NA, Hodge CC, Caudill SP, Brock JW, Needham LL \& Calafat AM 2004 Urinary levels of seven phthalate metabolites in the U.S. population from the National Health and Nutrition Examination Survey (NHANES) 1999-2000. Environmental Health Perspectives 112 331-338. (doi:10.1289/ehp.6723)

Skakkebaek NE, Rajpert-De Meyts E \& Main KM 2001 Testicular dysgenesis syndrome: an increasingly common developmental disorder with environmental aspects. Human Reproduction 16 972-978. (doi:10.1093/ humrep/16.5.972)

Skakkebaek NE, Rajpert-De Meyts E, Jørgensen N, Main KM, Leffers H \& Andersson AM 2007 Testicular cancer trends as 'whistle blowers' of testicular developmental problems in populations. International Journal of Andrology 30 198-204. (doi:10.1111/j.1365-2605.2007. 00776.x)
Sloboda DM, Hart R, Doherty DA, Pennell CE \& Hickey M 2007 Age at menarche: Influences of prenatal and postnatal growth. Journal of Clinical Endocrinology and Metabolism 92 46-50. (doi:10.1210/jc. 2006-1378)

Sloboda DM, Hickey M \& Hart R 2011 Reproduction in females: the role of the early life environment. Human Reproduction Update 17 210-227. (doi:10.1093/humupd/dmq048)

Suzuki Y, Yoshinaga J, Mizumoto Y, Serizawa S \& Shiraishi H 2012 Foetal exposure to phthalate esters and anogenital distance in male newborns. International Journal of Andrology 35 236-244. (doi:10.1111/j.13652605.2011.01190.x)

Swan SH, Main KM, Liu F, Stewart SL, Kruse RL, Calafat AM, Mao CS, Redmon JB, Ternand CL, Sullivan S et al. 2005 Decrease in anogenital distance among male infants with prenatal phthalate exposure. Environmental Health Perspectives 113 1056-1061. (doi:10.1289/ehp.8100)

Takai R, Hayashi S, Kiyokawa J, Iwata Y, Matsuo S, Suzuki M, Mizoguchi K, Chiba S \& Deki T 2009 Collaborative work on evaluation of ovarian toxicity. 10) Two- or four-week repeated dose studies and fertility study of di-(2-ethylhexyl) phthalate (DEHP) in female rats. Journal of Toxicological Sciences 34 (Suppl 1) SP111-SP119. (doi:10.2131/jts.34. S111)

Toppari J, Virtanen HE, Main KM \& Skakkebaek NE 2010 Cryptorchidism and hypospadias as sign of testicular dysgenesis syndrome (TDS): environmental connection. Birth Defects Research. Part A, Clinical and Molecular Teratology 88 910-919. (doi:10.1002/bdra.20707)

Uzumcu M, Kuhn PE, Marano JE, Armenti AE \& Passantino L 2006 Early postnatal methoxychlor exposure inhibits folliculogenesis and stimulates anti-Müllerian hormone production in the rat ovary. Journal of Endocrinology 191 549-558. (doi:10.1677/joe.1.06592)

Wang W, Craig ZR, Basavarajappa MS, Gupta RK \& Flaws JA 2012 Di (2-ethylhexyl) phthalate inhibits growth of mouse ovarian antral follicles through an oxidative stress pathway. Toxicology and Applied Pharmacology 258 288-295. (doi:10.1016/j.taap.2011.11.008)

Welsh M, Saunders PT, Fisken M, Scott HM, Hutchison GR, Smith LB \& Sharpe RM 2008 Identification in rats of a programming window for reproductive tract masculinization, disruption of which leads to hypospadias and cryptorchidism. Journal of Clinical Investigation 118 1479-1490. (doi:10.1172/JCl34241)

Wittassek M, Angerer J, Kolossa-Gehring M, Schafer SD, Klockenbusch W, Dobler L, Gunsel AK, Muller A \& Wiesmuller GA 2009 Fetal exposure to phthalates - a pilot study. International Journal of Hygiene and Environmental Health 212 492-498. (doi:10.1016/j.ijheh.2009.04.001)

Wolff MS, Teitelbaum SL, Pinney SM, Windham G, Liao L, Biro F, Kushi LH, Erdmann C, Hiatt RA, Rybak ME et al. 2010 Investigation of relationships between urinary biomarkers of phytoestrogens, phthalates, and phenols and pubertal stages in girls. Environmental Health Perspectives 118 1039-1046. (doi:10.1289/ehp.0901690)

Yuan K, Zhao B, Li XW, Hu GX, Su Y, Chu Y, Akingbemi BT, Lian QQ \& Ge RS 2012 Effects of phthalates on 3 $\beta$-hydroxysteroid dehydrogenase and $17 \beta$-hydroxysteroid dehydrogenase 3 activities in human and rat testes. Chemico-Biological Interactions 195 180-188. (doi:10.1016/ j.cbi.2011.12.008)

Zawadzki J \& Dunaif A 1992 Diagnostic criteria for polycystic ovary syndrome: towards a rational approach. In Polycystic Ovarian Syndrome, pp 374-388. Eds A Dunaif, JR Givens, F Haseltine, G R Merriam. Blackwell: Boston, MA, USA.

Received 23 July 2013

First decision 19 August 2013

Revised manuscript received 23 August 2013

Accepted 11 September 2013 